\title{
Continuous Blood Pressure Monitoring Method Based on Multiple Photoplethysmography Features
}

\author{
Yang XU', Zhipei HUANG ${ }^{\mathrm{a}, 1}$, Jiankang WU ${ }^{\mathrm{a}, \mathrm{b}}$ and Zhongdi LIU ${ }^{\mathrm{a}}$ \\ ${ }^{a}$ School of Electronic, Electrical and Communication Engineering, University of \\ Chinese Academy of Sciences, Beijing, China \\ ${ }^{\mathrm{b}}$ CAS Institute of Healthcare Technologies, Nanjing, China
}

\begin{abstract}
Continuous blood pressure monitoring is of great significance for the prevention and early diagnosis of cardiovascular diseases. However, the existing continuous blood pressure monitoring methods, especially the sleeveless blood pressure monitoring methods, are complex and computationally heavy. In this paper, we propose a method, using plethysmography (PPG) signals alone, to estimate continuous blood pressure by extracting multiple PPG features related to intravascular blood flow characteristics. The performance of our method was evaluated using ten minutes synchronized PPG signals and continuous blood pressure from 21 healthy volunteers and 19 patients with hypertension and diabetes. The test results have shown that the absolute mean errors and standard deviation errors between the estimated and referenced blood pressure are $3.22 \pm 0.66 \mathrm{mmHg}$ for systolic blood pressure and $2.11 \pm 1.11 \mathrm{mmHg}$ for diastolic blood pressure, which meet AAMI (the association for the advancement of medical instrumentation) error acceptance.
\end{abstract}

Keywords. Continuous blood pressure monitoring, plethysmography (PPG) features, pulse transit time (PTT), slope transit time (STT)

\section{Introduction}

As an increasing leading cause of deaths, cardiovascular disease (CVD) has been the focus of medical research. Blood pressure (BP) is one of the most important risk factors of CVD. Hence continuous BP measurement and monitoring is highly significant in all healthcare practices, including medical check, diagnosis and operation. The golden standard continuous BP measurement in current medial society is invasive, by inserting a pressure sensor into artery blood vessel [1]. This invasive method is only used in certain critical cases, not suitable for ordinary medical cases and public usage. Therefore, recent studies have aimed to propose a non-invasive method to obtain accurate continuous blood pressure. Some researchers have focused in the ultrasound-based method, which detect the changes in vessel diameter in arteries through the Doppler effect. However, this method has a rather high sensor cost and may have a huge impact in the life of the users [2]. Another indirect monitoring method, the volume-compensation method, has

1 Corresponding Author, Zhipei HUANG, School of Electronic, Electrical and Communication Engineering, University of Chinese Academy of Sciences, Beijing, China; Email: zhphuang@ucas.ac.cn. 
been shown to be stable and accurate. Nonetheless, the main disadvantage of this method is that long-term continuous measurements may lead to venous congestion and cause discomfort for the patients [3]. The arterial tonometry method has the advantage of high measurement accuracy and basically enables longer-term noninvasive continuous blood pressure measurements, but the elasticity of the subject's skin and the position of the sensor can affect the accuracy [4]. To address the problems of high cost, difficulty of use, and uncomfortable experience of patient, some researchers have turned to the use of pulse transit time (PTT) as an indicator to indirectly estimate blood pressure, which is the time required for the pulse pressure (PP) waveform to propagate through the arterial tree [5].

Recent PTT-based method studies focused on the improvement of estimation accuracy limitations, mainly because the most important parameter in PTT-based methods, pulse transit time, cannot be detected directly [6]. A substitute parameter called pulse arrival time (PAT) is usually used to replace PTT in BP estimation. This substitution brings a lot of problems. On the one hand, as shown in figure 1a, the pulse arrival time is measured from a feature point (point R) in the ECG of the cardiac cycle and a feature point (point F) in the PPG, which means that both PPG and ECG need to be recorded simultaneously and feature points need to be detected accurately. On the other hand, ECG-PPG-based pulse arrival time PAT contains not only PTT but also preejection period, which includes left ventricular electromechanical delay time and isovolumic contraction cycle. Direct use of PAT will seriously affect the accuracy of PTT-based blood pressure estimation [7, 8]. These problems hinder the popularity of PTT-based blood pressure estimation, and alternative ways to eliminate these effects need to be found.

Yang et al proposed a single PPG signal feature: mean slope transit time (MSTT) [9], which is a new and more stable feature than slope transit time (STT). However, the claimed good performance may drift as the result of the facts that only healthy people and young people participated the experiments and single PPG feature based BP estimation model might be sensitive to signal quality.

Here we attempt to develop a continuous blood pressure estimation method from PPG only, referred to as PPG-BP. The main contribution in PPG-BP include: First, three blood pressure related feature measures from PPG, namely, the height slope transit time (NSTT), point of minimum derivative in descending branch derivative (PMDD) and the peak of tidal wave (PTW), were extracted from PPG signal. Second, invariant feature extraction algorithms have been carefully designed to reduce the effect of variance of sensor performance and interferences.

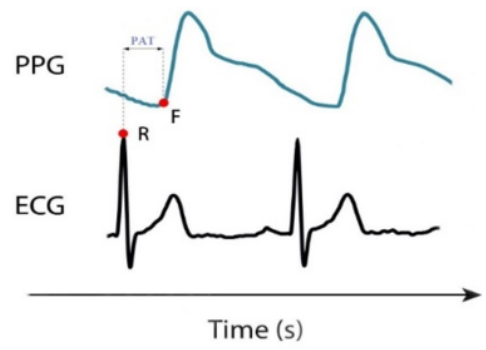

(a)

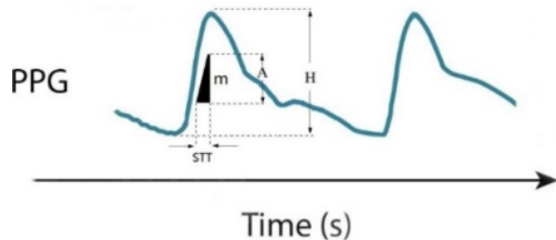

(b)

Figure 1. Schematic of Measurement of (a) PAT and (b) STT. 
In the rest of this paper, the physiological basis of three feature measures is discussed in section 2. Section 3 describes the extraction of feature points, the BP estimation model and implementation. Finally, the experimental results are discussed in Section 4 and the conclusions are explained in Section 5.

\section{Physiological Basis of Features Selections}

Many factors affect the transmission of pulse waves in arteries, such as arterial diameter and elasticity. Paul reported that by observing the changes of pulse waves during the respiratory cycle, the upward slope of the initial pulse wave component is proportional to the transit time, which may be a potential alternative to PTT in hemodynamic studies. This rate of increase is not affected by the heart rate and ejection time [10], which means that it will provide a more accurate alternative to PTT than other PAT. In this case, Paul proposed a new PPG factor-slope transit time (STT) as an alternative to PTT, which is calculated from a single base point upstream of PPG, and the slope transit time (STT) is illustrated in figure $1 \mathrm{~b}$.

In the study of Addison [10], STT was defined as equation (1):

$$
S T T=\frac{A}{m}
$$

where $m$ is the max-slope of upslope of PPG, and $A$ is the constant related to the amplitude of PPG.

In this study, we proposed a feature measure called Normalized Slope Transit Time (NSTT) as a proxy alternative of PTT. NSTT refers to the slope transit time, but improves its stability with normalization by PPG height, to reduce the variation among individual samples due to sensors and other interferences. We define NSTT as equation (2):

$$
N S T T=\frac{H}{m}=\frac{H}{\max (\Delta A / \Delta t)}
$$

where $m$ is the max-slope of upslope of PPG, $H$ is the amplitude range of PPG. $\Delta A$ is proportional to $H$, thus, NSTT is invariant to PPG signal amplitude, which is normally sensitive to sensor and its worn manner.

The accuracy of using a single feature to estimate continuous blood pressure is often poor. In addition to individual differences and the influence of different devices, the shape of PPG waveform is also affected by many factors, including contact force, ambient temperature, drugs, vascular movement, arteriosclerosis, postural changes and so on. Therefore, the use of NSTT alone can't fully reflect the change of blood pressure.

A complete PPG waveform is consisted of ascending part and descending part, as shown in figure 2, where $\mathrm{F}$ is the valley of the pulse wave, $\mathrm{A}$ is the peak, $\mathrm{B}$ is the tidal wave peak, $\mathrm{C}$ is the dicrotic wave foot, and $\mathrm{D}$ is the dicrotic notch. The FA segment is an ascending branch, which is caused by the rapid increase of blood flow during left ventricular ejection. The slope and amplitude of the ascending branch are affected by ejection velocity, cardiac output and ejection resistance. During the late ejection and diastole, the blood flow into the artery decreased, forming the descending branch of AF'. In the descending branch, the blood flow does not decrease monotonously, the sudden increase of vascular resistance caused by the blood entering the branch artery will lead 
to the emergence of tidal wave, while the reflected wave caused by blood hitting the heart valve forms the repulse wave [11].

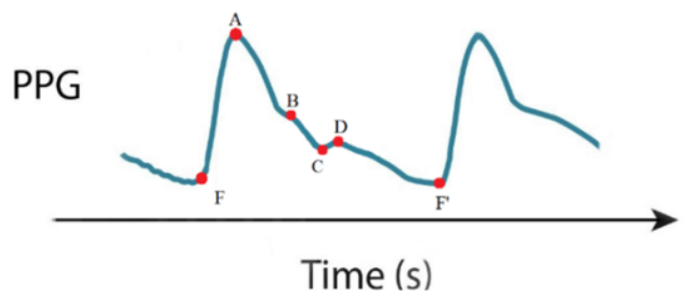

Figure 2. PPG waveform and feature points.

Two new PPG morphological feature measures were introduced in our BP estimation algorithm, Point of Minimum Derivative in Descending Branch Derivative (PMDD) and the Peak of Tidal Wave (PTW). Pulse wave could be regarded as combination of percussion wave and reflected wave [12]. Percussion wave is caused from LV ejection that increases the arterial wall linearly. Reflected wave contains two parts, one is tidal wave, reflected from the small artery. The other is dicrotic wave, reflective oscillatory wave occurred from the blood crash into aortic valve by blood pressure of aorta.

Assuming other arterial parameters remains constant and BP increases, pulse wave velocity (PWV) will increase, which means pulse wave transfer faster [13, 14]. Hence the reflected wave will meet percussion wave sooner, which enhances the fusion of two waves. The circumstance above will influence the shape of PPG as followings. Tidal wave will be closer to peak point and its amplitude will be raised. It means the increase of PTW. For dicrotic wave, it is also closer to percussion wave and the position of its descending part will be earlier in cardiac cycle than before, which leads to the decrease of PMDD (figure 3).

As can be seen from above, PMDD and PTW could reflect the variation of PWV and further indicate the changes of BP. These two feature measures were added into our BP estimation algorithm to improve accuracy.

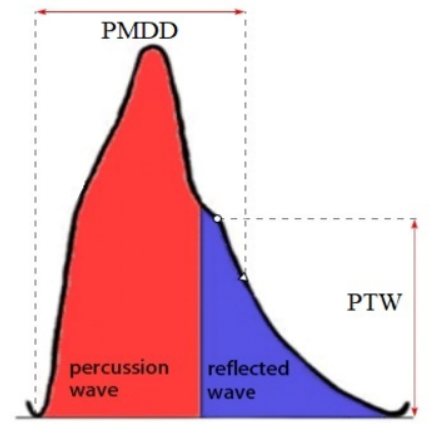

(A)

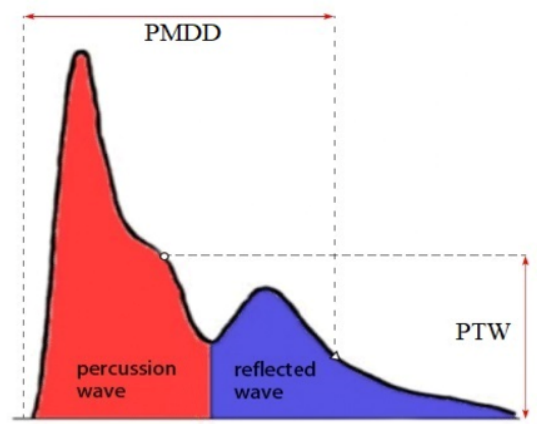

(B)

Figure 3 PMDD and PTW in PPG waveform. Blood pressure of (A) is higher than (B).

As shown in figure 4, PTW is defined as the ratio of the height difference between tidal wave (point B) and point F of PPG to the height of pulse wave $\left(H=h_{A}-h_{F}\right)$, which can be calculated as equation (3). 


$$
P T W=\frac{h_{B}-h_{F}}{H}
$$

PMDD was defined as the time interval between the minimum point of the derivative of descending branch (D point) and F point of PPG, and PMDD could be calculated as equation (4).

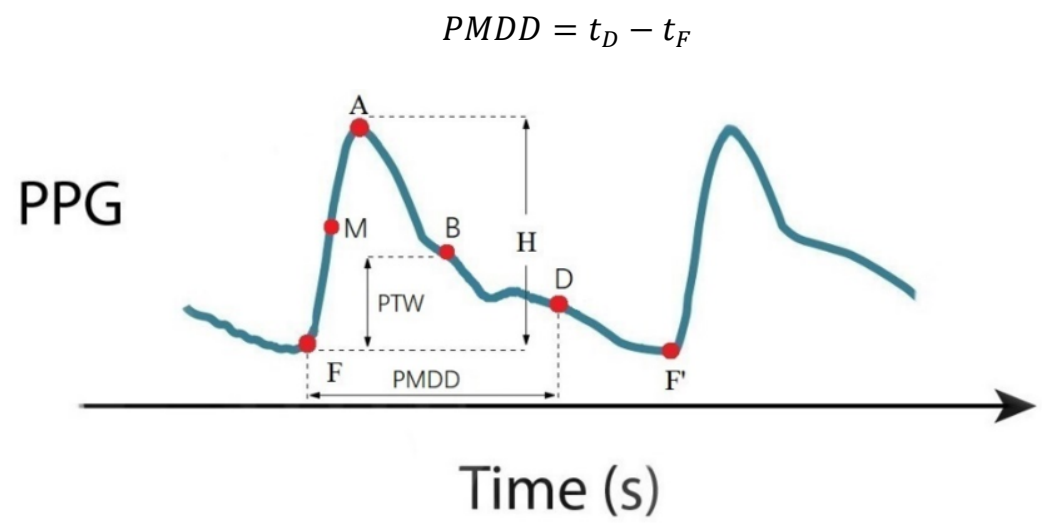

Figure 4. Illustration of feature points and PMDD and PTW definition in PPG signal.

\section{Methods}

\subsection{Data Acquisition}

In our experiment, the Finometer ${ }^{\circledR}$ Pro (Finapres Medical System, The Netherlands, a non-invasive continuous blood pressure monitor certified by FDA) was used to monitor noninvasive beat-to-beat blood pressure as reference. At the same time, in order to compare with the traditional PTT method, PPG and ECG signal were collected by a physiological signal synchronous collecting device developed by ourselves. Two ECG electrodes were placed on left and right chest, and PPG sensor was worn on left middle finger. The physiological signals were sampling by $1 \mathrm{kHz}$ and 16 bits resolution. The synchronization error between PPG and ECG signal is no more than $1 \mathrm{~ms}$.

Twenty-one healthy volunteers (aged $45 \pm 20$ years old) and 19 patients with hypertension and diabetes (aged $54 \pm 15$ years old) were recruited. The volunteers were asked to lie in the hospital bed for 10 minutes, keep resting during the whole process, and record PPG, ECG and blood pressure signals continuously at the same time.

\subsection{Extraction of Feature Points}

There are 5 feature points to be extracted (figure 5). The A point is the maximum point in the cardiac cycle. The M point is the maximum slope point of PPG uphill. The F point can be extracted by finding the minimum point on the left side of the PPG peak. The B point was detected using Mallet algorithm and the $\mathrm{D}$ point was extracted as minimum point of the derivative of the part between A point and F' point in next cardiac cycle. 


\subsection{BP Estimation Model}

Because most studies in this field use linear relationship, which has strong universality, stable performance and simple calculation, this study will use linear relationship for follow-up research [15]. PTT-based BP estimation algorithm as equations (5) and (6):

$$
\begin{aligned}
& S B P=a P T T+b \\
& D B P=c P T T+d
\end{aligned}
$$

where $S B P$ was systolic pressure, $D B P$ was diastolic pressure, $a, b, c$ and $d$ are constant parameters specific to each subject, and should be initialized and calibrated at the beginning of BP measurement and monitoring.

A modified PTT-based BP estimation algorithm using NSTT, PTW and PMDD was developed as equations (7) and (8).

$$
\begin{aligned}
& S B P=a_{0} N S T T+a_{1} P M D D+a_{2} P T W+a_{3} \\
& D B P=b_{0} N S T T+b_{1} P M D D+b_{2} P T W+b_{3}
\end{aligned}
$$

where $S B P$ was systolic pressure, $D B P$ was diastolic pressure, $a_{0}, a_{1}, a_{2}, a_{3}, b_{0}, b_{1}$, $b_{2}$ and $b_{3}$ are personalized constant parameters.

\subsection{Parameters Initialization}

Least square method was used to initialize parameters as equations (9)-(11) and (14).

$$
\begin{gathered}
X=\left[\begin{array}{cccc}
N S T T_{1} & P M D D_{1} & P T W_{1} & 1 \\
N S T T_{2} & P M D D_{2} & P T W_{2} & 1 \\
\ldots & & \\
N S T T_{n} & P M D D_{n} & P T W_{n} & 1
\end{array}\right] \\
Y=\left[\begin{array}{cc}
S B P_{1} & D B P_{1} \\
S B P_{2} & D B P_{2} \\
\ldots & \ldots \\
S B P_{n} & D B P_{n}
\end{array}\right] \\
\theta=\left(X^{T} X\right)^{-1} X^{T} Y
\end{gathered}
$$

where value with $n$ subscript represented the corresponding value in the $n$th initialization sample and $\theta$ was defined as equation (12). Generally, $n$ could be 4 to 10 .

$$
\theta=\left[\begin{array}{ll}
a_{0} & b_{0} \\
a_{1} & b_{1} \\
a_{2} & b_{2} \\
a_{3} & b_{3}
\end{array}\right]
$$




\section{Results and Discussion}

\subsection{Correlations of Different Feature Measures to BP}

The correlation coefficients of a group of indicators to SBP/DBP across all the subjects were calculated as table 1. Feature measure to be compared contained heart rate (HR) and PAT, a widely concerned in recent years. It was observed that and NSTT PTW showed a positive correlation with SBP/DBP and PMDD did the opposite, both of which achieved higher correlation coefficients than HR and PAT.

Table 1. Mean \pm SD of the correlation coefficients of features measure (NSTT, PTW, PMDD, HR, PAT) to SBP/DBP.

\begin{tabular}{llllll}
\hline & NSTT & PTW & PMDD & HR & PAT \\
\hline SBP & $0.59 \pm 0.18$ & $0.53 \pm 0.42$ & $-0.39 \pm 0.26$ & $0.30 \pm 0.29$ & $-0.38 \pm 0.42$ \\
DBP & $0.49 \pm 0.15$ & $0.45 \pm 0.39$ & $-0.35 \pm 0.27$ & $0.23 \pm 0.24$ & $0.30 \pm 0.39$ \\
\hline
\end{tabular}

According to table 1, the variance of correlation between PTW and SBP/DBP was obviously larger than other features measure, mainly because the difficulty and inaccuracy of PTW's extraction.

\subsection{Performance of BP Estimation Model}

Our proposed method, PTT-based method and MSTT-based method [9] were all implemented and evaluated across our dataset. Figure 5 shows one segment sample of SBP estimation results for hypertensive patient. The estimation errors are presented in table 2. with absolute mean and standard deviation (SD). Comparing with PTT-based method and MSTT-based method, our modified method achieved an apparent decrease of absolute mean and SD errors.

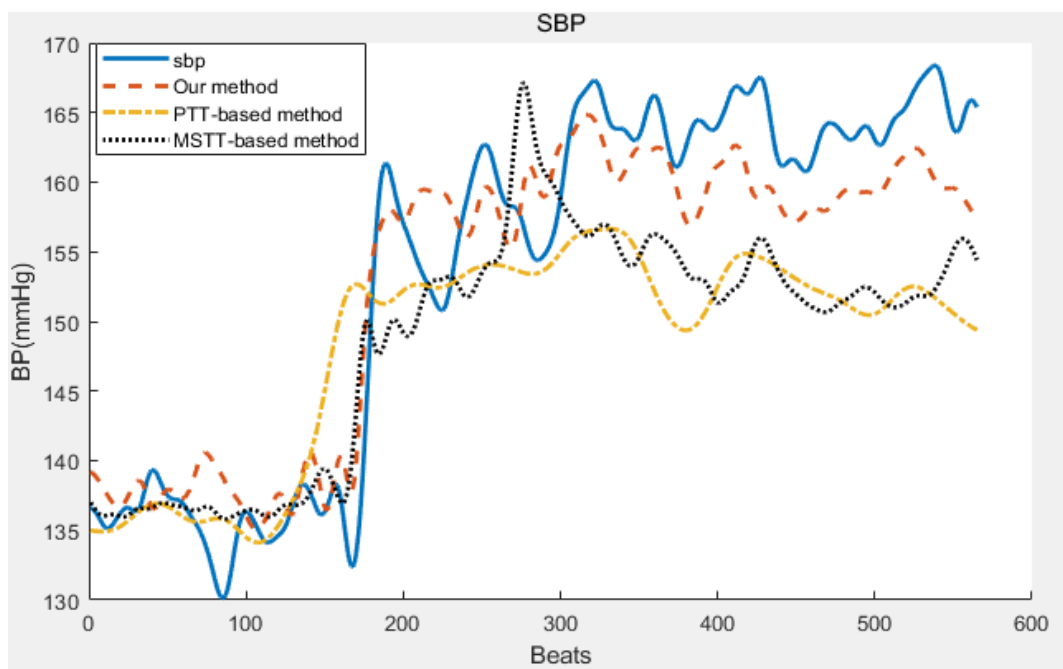

Figure 5. Reference SBP (blue), estimated SBP by our method (red), PTT-based method (yellow) and MSTTbased method (black). 
Table 2. Absolute error (mean \pm sd.) of three bp estimation methods.

\begin{tabular}{|c|c|c|c|}
\hline & \multicolumn{3}{|l|}{ Methods } \\
\hline & Our method & PTT-based method & MSTT-based method \\
\hline DBP & $2.11 \pm 1.11$ & $3.22 \pm 4.55$ & $2.88 \pm 3.76$ \\
\hline SBP & $3.22 \pm 0.66$ & $4.82 \pm 5.07$ & $3.97 \pm 3.26$ \\
\hline
\end{tabular}

British Hypertension Society (BHS) standard [16] and Advancement of Medical Instrumentation (AAMI) [17] standard is used to evaluate our method as tables 3 and 4 respectfully. It is obvious that our BP estimation method had a satisfying performance.

Table 3. Evaluation using BHS standard.

\begin{tabular}{lllllll}
\hline & SBP & \multicolumn{7}{c}{ DBP } \\
\cline { 2 - 7 } & A & B & C & A & B & C \\
\cline { 2 - 7 } Num. & 26 & 38 & 40 & 37 & 40 & 40 \\
Per. & $65.0 \%$ & $98.0 \%$ & $100.0 \%$ & $92.5 \%$ & $100.0 \%$ & $100.0 \%$ \\
\hline
\end{tabular}

Table 4. Evaluation using AAMI standard.

\begin{tabular}{llll}
\hline SBP & & DBP & \\
\hline Num. & Per. & Num. & Per. \\
\hline 29 & $72.5 \%$ & 35 & $87.5 \%$ \\
\hline
\end{tabular}

\section{Conclusion}

This paper presents a method to estimate BP by extracting the features measure of NSTT, PTW and PMDD using PPG signals. Compared with the previously proposed PTT-based method, our method shows better accuracy. This method is not affected by heart rate effect and pre-ejection period, and is simple and linear. This method has the potential to be applied to continuous blood pressure measurement. There are still some limitations in this study. The BP estimation model for NSTT is only a modification to the PTT-based model, and it may not be the most suitable model for NSTT. In future research, we may conduct further research to find a more accurate blood pressure estimation model.

\section{References}

[1] Silver, M.J., et al., Evaluation of a new blood-conserving arterial line system for patients in intensive care units. Critical care medicine, 1993. 21(4): p. 507-511

[2] Kazamias, T.M., et al., Blood pressure measurement with Doppler ultrasonic flowmeter.Journal of Applied Physiology, 1971. 30(4): p. 585-588

[3] Tanaka, S. and K. Yamakoshi, Ambulatory instrument for monitoring indirect beat-to-beat blood pressure in superficial temporal artery using volume-compensation method. Medical and Biological Engineering and Computing, 1996. 34(6): p. 441-447.

[4] Saugel, B., et al., The T-Line TL-200 system for continuous non-invasive blood pressure measurement in medical intensive care unit patients. Intensive care medicine, 2012. 38(9): p.1471-1477.

[5] Peter, L., N. Noury, and M. Cerny, A review of methods for non-invasive and continuous blood pressure monitoring: Pulse transit time method is promising? Irbm, 2014. 35(5): p. 271-282

[6] Li, Y., S. Li, and N. Deng, Noninvasive and continuous blood pressure monitoring with better accuracy by combining pulse arrival time and peak delay. International Journal of Distributed Sensor Networks, 2018. 14(12): p. 1550147718818738. 
[7] G. Zhang, A. C. Cottrell, I. C. Henry, and D. B. McCombie, Assessment of pre-ejection period in ambulatory subjects using seismocardiogram in a wearable blood pressure monitor, in 2016 38th Annual International Conference of the IEEE Engineering in Medicine and Biology Society (EMBC), 2016, pp. 3386-3389.

[8] Q. Liu, B. P. Yan, C. M. Yu, Y. T. Zhang, and C. C. Y. Poon, Attenuation of Systolic Blood Pressure and Pulse Transit Time Hysteresis During Exercise and Recovery in Cardiovascular Patients, IEEE Transactions on Biomedical Engineering, vol. 61, no. 2, 2014, pp. 346-352.

[9] Y. Chen, S. Cheng, T. Wang, and T. Ma, Novel blood pressure estimation method using single photoplethysmography feature, in Engineering in Medicine and Biology Society (EMBC), 2017 39th Annual International Conference of the IEEE. IEEE, 2017, pp. 1712-1715.

[10] P. S. Addison, Slope transit time (stt): A pulse transit time proxy requiring only a single signal fiducial point, IEEE Transactions on Biomedical Engineering, vol. 63, no. 11, 2016, pp. 2441-2444.

[11] LUO Zhichang, ZHANG Song, YANG Wenming, et al. The research about pulse wave characteristic information, in Journal of Beijing University of Technology 1996, 22(1): 71-79

[12] OCHI H, SHIMADA T, EFFECT OF A DECREASE IN AORTIC COMPLIANCE ON THE ISOVOLUMIC RELAXATION PERIOD OF THE LEFT VENTRICLE IN MAN in American Journal of Noninvasive Cardiology, vol 5, 1991, P:148-154

[13] Remington, J.W., The physiology of the aorta and major arteries, in Handbook of physiology.1963, American Physiological Society Washington, DC. p. 808.

[14] Hughes, D., et al., Measurements of Young's modulus of elasticity of the canine aorta with ultrasound. Ultrasonic Imaging, 1979. 1(4): p. 356-367.

[15] Rastegar S, Gholam Hosseini H, Lowe A. Non-invasive continuous blood pressure monitoring systems: current and proposed technology issues and challenges. Australas Phys Eng Sci Med. 2019; 43:11-28.

[16] O'Brien, E., et al., The British Hypertension Society protocol for the evaluation of automated and semiautomated blood pressure measuring devices with special reference to ambulatory systems. Journal of hypertension, 1990. 8(7): p. 607-619

[17] White, W.B., et al., National standard for measurement of resting and ambulatory blood pressures with automated sphygmomanometers. Hypertension, 1993. 21(4): p. 504-509. 